\title{
LncRNA HOTAIR promotes proliferation and inhibits apoptosis by sponging miR-214-3p in HPV16 positive cervical cancer cells
}

\section{Yu Zhou}

Wuhan University School of Basic Medical Sciences

\section{Yuqing Wang}

Wuhan University School of Basic Medical Sciences https://orcid.org/0000-0002-9548-2051

\section{Mingying Lin}

Wuhan University Second Clinical College: Wuhan University Zhongnan Hospital

\section{Daiqian Wu}

Wuhan University Second Clinical College: Wuhan University Zhongnan Hospital

Min Zhao ( $\nabla$ minzhao@whu.edu.cn )

Wuhan University https://orcid.org/0000-0001-9207-4629

\section{Primary research}

Keywords: cervical cancer, HPV16 E7, HOTAIR, miR-214-3p, ceRNA

Posted Date: March 18th, 2021

DOl: https://doi.org/10.21203/rs.3.rs-299026/v1

License: (c) (i) This work is licensed under a Creative Commons Attribution 4.0 International License. Read Full License

Version of Record: A version of this preprint was published at Cancer Cell International on July 28th, 2021. See the published version at https://doi.org/10.1186/s12935-021-02103-7. 


\section{Abstract \\ Background}

Cervical cancer (CC) is one of the most common gynecological malignancies all around the world. The mechanisms of cervical carcinoma formation remain under close scrutiny. The long non-coding RNAs (IncRNA) and microRNAs (miRNAs) play important roles in controlling gene expression and promoting the development and progression of cervical cancer by acting as competitive endogenous RNA (ceRNA). However, the roles of IncRNA associated with ceRNAs in cervical carcinogenesis remains unknown. In this study, the expression of LncRNA HOTAIR was investigated in HPV16 positive cervical cancer cells, the candidate miRNAs and target genes were identified to clarify putative ceRNAs of HOTAIR/miRNA in cervical cancer cells.

\section{Methods}

The proliferate ability of cells was measured by CCK8 and EdU incorporation assays and cell apoptosis was analyzed by flow cytometry. The expression of HOTAIR, miR-214-3p, HPV16 E7 mRNA were detected by qRT-PCR. As for searching for the interaction between miR-214-3p and HOTAIR, the binding sites for miR-214-3p on HOTAIR was predicted by starbase v2.0 database, then dual-luciferase assay was used to verify the binding sites. In addition, Gene Ontology (GO) and protein-protein interaction (PPI) network analysis of target genes of miR-214-3p were performed with bioinformatics analysis. For potential signaling pathway regulated by miR-214-3p, we conducted pathway enrichment analysis by KEGG analysis and obtained key pathways in cervical cancer cells.

\section{Results}

Our results showed that the expression of HOTAIR was up-regulated, while that of miR-214-3p was downregulated in HPV16-positive cervical cancer cells. The expression status of HPV16 E7 played an important role in regulating the expression of HOTAIR or miR-214-3p in cervical cancer cells. LncRNA HOTAIR knockdown could significantly inhibited cell proliferate ability and promote cellular apoptosis, whereas the inhibition of miR-214-3p expression partially reversed such results. Bioinformatics analysis identified 1451 genes as target genes of miR-214-3p. The Gene ontology (GO) and KEGG Pathway enrichment analysis showed that these target genes were mainly related to regulation of cell communication, protein binding, enzyme binding and transferase activity, and Wnt ligand biogenesis. Pathway enrichment analysis results showed that the predicted target genes were significantly enriched in Wnt/ $\beta$-catenin signaling pathway. Finally, our results confirmed that miR-214-3p could significantly inhibit $\beta$-catenin expression in HPV16 positive cancer cells by qPCR and WB analysis.

\section{Conclusion}


HOTAIR could act as a ceRNA through binding to miR-214-3p, promote cell proliferation and inhibit the apoptosis of HPV16 positive cervical cancer. HOTAIR/miR-214-3p/Wnt/ $\beta$-catenin signaling pathway might play important roles related with HPV16 positive cervical cancer. Our results provided a new perspective for identifying novel biomarkers for cervical cancer.

\section{Introduction}

Cervical cancer is one of the most common gynecological malignancies in worldwide, with an estimated 528,000 new cases and 311,000 deaths worldwide each year, more than any other gynecologic tumor[1]. High-risk HPV (HR-HPV) infection is the main risk factor for cervical cancer, and $95 \%$ of cases are caused by persistent infections with HR-HPV [2]. As is known to all, the integration of HR-HPV DNA into the host cell genome resulting in persistent overexpression of HPV E6 and E7 oncoproteins, subsequently induce immobilization of cells through their inhibitory effects on the tumor suppressor proteins p53 and pRb, respectively $[3,4]$. In the recent years, the morbidity and mortality of cervical cancer have been significantly reduced owing to the availability of effective prophylactic vaccines against the most important carcinogenic HPV types including Gardasil and Cervarix[5]. However, although early cervical cancer could be treated with surgery or radiation, the prognosis of advanced cervical cancer patients generally remains poor, and the metastatic cervical cancer is incurable yet and the overall 5 years' survival rate is only approximately $40 \%$ after conventional treatments are used[6]. In addition, the steps and mechanisms of the progression from preneoplastic lesions, cervical intraepithelial neoplasia (CIN) to carcinoma remain unknown. Therefore, the further exploring the mechanism underlying and new therapeutic approaches are urgently required.

Although the persistent infections with HR-HPV was the main risk factor for cervical cancer, most HPV infections could be cleared spontaneously in immuno-competent subjects, so only a minority of women infected with HR-HPV will ultimately develop cervical cancer [7]. Therefore, additional genetic event must have contributed to the initiation and ultimate progression of the cervical cancer. In the recent years, the omics-based studies have revealed novel molecular mechanisms leading to cervical carcinogenesis. Epigenetic modifications, including deregulation of microRNA (miRNA), long non-protein coding RNA (IncRNA) and circular RNA (circRNA), have shown to play important roles in cell transformation during distinct stages of cervical intraepithelial neoplasia and cervical carcinoma development. LncRNAs were regulatory transcripts longer than 200 nucleotides, including a 5' 7-methylguanosine cap and a 3' poly (A) tail similarly to messenger RNAs [8]. The function of IncRNA mainly through cis and trans mode to regulate the gene transcription and post transcriptional modification of target genes $[9,10]$. It is generally thought that IncRNAs regulate downstream target genes through competitive endogenous RNA (ceRNA) mechanisms. This means that IncRNA could regulate the expression of gene through competing binding to miRNAs at the post-transcriptional level. For example, IncRNAs regulated target genes transcription by interacting with chromatin-modifying complexes at specific regulatory regions, or as a molecular sponge of transcription factors and miRNAs[11]. Numerous experimental results confirm that IncRNAs could play a crucial role in the development of cervical cancer. Around 14 IncRNAs have shown to be altered in cervical carcinoma affecting important metabolic pathways such as PI3K/AKT, Notch signaling and 
MAPK pathway[12]. Moreover, some IncRNAs, including MALAT1, CCEPR, and TMPOP2, could interact with HPV16 E6 and E7 oncogene to enhance its carcinogenesis effect in the development of cervical neoplasia $[13,14]$.

The IncRNA HOTAIR (HOX transcript antisense intergenic RNA) was the first to be found trans regulatory long non-coding RNA, encoded by the antisense strand of the HOXC gene located in the chromosome 12 q13.13 [15]. The HOTAIR 5' domain could bind the zeste homolog 2 (EZH2) and to silence the target gene nemo-like kinase (NLK) [16]. Recently, HOTAIR has been conformed as molecular sponge for several miRNAs in various malignant tumors including colorectal cancer, hepatocellular carcinoma, pancreatic carcinoma and cervical cancer [17-19]. However, the precise role and function of HOTAIR in cervical cancer remained unclear, only a relatively small number of studies have associated HOTAIR with cervical cancer. The current research shows that HOTAIR was highly expressed and could be used as an indicator of poor prognosis of cervical cancer [20,21]. A recent study has revealed that HPV16 E7 inhibited the recruitment of the PRC2 complex and promoted the development of cervical cancer by competing the binding site of the PRC2 complex on HOTAIR [22]. HOTAIR could regulate the miR-143-3p/BCL2 axis and miR-23b/MAPK1 axis contributing to cell proliferation and metastasis[23, 24]. These researches indicated that HOTAIR might play an important role in cervical cancer. However, the relevant research results were still very limited in cervical cancer.

Numerous studies have analyzed the miRNAs expression to identify significant variations during the transition from low to high grade cervical neoplasia and to invasive cervical cancer[25, 26]. Many miRNAs (let-7b, let-7c, miR-21, miR-23b, miR-196b, miR-143, miR-126-3p, miR-20b-5p, miR-451a, and miR-144-3p) were identified as potential biomarkers for cervical cancer diagnosis, prognosis and cancer stage, even have been shown effective for diagnosis of cervical adenocarcinoma and high grade intraepithelial lesions[27, 28, 29]. miR-214-3p was a miRNA involved in many cancer, including lung cancer, breast cancer, colon cancer, endometrial carcinoma, and pancreatic cancer in humans[30, 31]. In cervical cancer, miR-214-3p has been found to play an important role in regulating cell proliferation, apoptosis, cell invasion, metastasis and angiogenesis $[32,33]$. Unfortunately, although the potential effectiveness was identified in cervical cancer, the mechanism and status of miR-214-3p involve in ceRNA network in cervical cancer cells needs to be addressed. Moreover, there was no report about the correlation between IncRNA HOTAIR and miR-214-3p in cervical cancer cells so far. Therefore, more investigations were needed in order to deep research the interaction between IncRNA HOTAIR and miR-214-3p in cervical cancer.

In this study, we detected the expression of HOTAIR and miR-214-3p in cervical cancer cells, conducted the target genes and pathway enrichment analysis with bioinformatics analysis. The effects of HOTAIRmiR-214-3p ceRNA network was specifically investigated to clarify the mechanism and potential target genes of cervical carcinogenesis.

\section{Materials And Methods}




\section{Cell culture and reagents}

The human cervical cancer cell lines SiHa, C-33A and immortalized cervical epithelial End1/E6E7 cells (ATCC, Rockville, MD) were all cultured in DMEM (Hyclone) supplemented with $10 \%$ fetal bovine serum (FBS, Gibco) and $1 \%$ penicillin/streptomycin cells at $37^{\circ} \mathrm{C}$ in a humidified $5 \% \mathrm{CO}_{2}$ atmosphere. All the consumables for cell culture were from JET BIOFIL ${ }^{\circledR}$ (Guangzhou, China). Primary antibodies against elF4E (C46H6, rabbit mAb, \#9742), $\beta$-catenin (D10A8, rabbit mAb, \#8480), goat peroxidase (HRP)conjugated secondary antibodies (\#7074) and antibody against $\beta$-actin (D6A8, rabbit mAb, \#8457) were all purchased from Cell Signaling Technology, China.

\section{RNA oligos and plasmid}

All RNA oligos including small interference RNAs (siRNAs), miRNA mimics, negative control (NC) and inhibitor were chemically synthesized by Shanghai GenePharma Company (Shanghai, China). Two siRNAs for HOTAIR were designed: si-HOTAIR-1 and si-HOTAIR-2, the better siRNA would be used in final experiment. In this study, cells were transfected with $160 \mathrm{nM}$ of siRNAs and negative control, and the final used concentration of miR-214-3p and mimics negative control was $80 \mathrm{nM}$. The following sequences of RNA oligos were used: si-HOTAIR-1 (GAGGCGCUAAUUAAUUGAUTTAUCAAUUAAUUAGCGCCUCTT), siHOTAIR-2 (GCA CAGAGCAACUCUAUAATTUUAUAGAGUUGCUCUGUGCTT), Si-NC (UUCUCCGAACGU GUCACGUTTACGUGACACGUUCGGAGAATT); miR-214-3p mimics (ACAGCAGGCACAG ACAGGCAGUUGCCUGUCUGUGCCUGCUGUUU), mimics NC (UUGUACUACACAAAAG UACUG). The shRNA for HPV16E7 was purchased from Genepharma (Suchow, China). The expression plasmid of HPV16 E7, PLXSN-E7 was preserved in our laboratory. In addition, we constructed the expression plasmid of HOTAIR functional motif, pEGFP-C1-HOTAIR-A, which contained the binding sites of miR-214-3p. The PCR amplification fragment of HOTAIR functional motif was insert between Mlul and Hind III sites on the EGFP-C1 vector.

\section{Dual luciferase assay}

For structure of of HOTAIR luciferase reporter plasmid, we firstly predicted the binding sites of miR-214-3p on HOTAIR by bioinformatics analysis (starbase v2.0 database), then the fragment of 3 '-UTR of HOTAIR containing miR-214-3p binding sites was amplified by PCR and inserted it into PMIR-REPORT ${ }^{\mathrm{TM}}$ Luciferase $^{2}$ vector. Cell transfection was performed with Lipofectamine 2000 regent (Invitrogen, U.S.A.). Then, luciferase reporter plasmid, renilla luciferase plasmid and miR-214-3p mimics/inhibitor were cotransfected into cells. After $24 \mathrm{~h}$ transfection, the luciferase activities were measured with a dual luciferase reporter assay system (Promega, Madison, U.S.A.) according to the manufacturer's instructions. Renilla luciferase intensity was normalized to firefly luciferase intensity.

\section{RNA isolation and qRT-PCR}

Total RNA was extracted with TRIzol reagent (Invitrogen). M-MLV reverse transcriptase (Promega, USA) and OligodT(15) were used to convert RNA into CDNA. Then quantitive RT-PCR was adopted to detect the related expression of HPV16 E7, HOTAIR, miR-214-3p and $\beta$-catenin. U6 snRNA was used for normalized 
of miR-213-3p, while the others were normalized to GAPDH. qRT-PCR analysis was performed with SybrGreen qPCR Mastermix (DBI® Bioscience, Germany) following the manufacturer's protocol. All the primers for PCR were shown in Table 2.

\section{Cell viability and proliferative capacity}

Cells were cultured on a 96-well plate and transfected with siRNAs or mimics for various times. Cell viability was then measured by the CCK-8 kit (Beyotime Biotechnology, Shanghai, China) according to the manufacturer's instructions. For CCK8 assay, 3000 per well cells were seeded into 96 -well plates $24-48 \mathrm{~h}$ after transfection and the absorbance were detected by microplate reader at $450 \mathrm{~nm}$. Cell proliferation capacity was evaluated by 5-Ethynyl-2'-deoxyuridine (EdU) incorporation assay. EdU is a thymidine analogue that can be inserted into the replicating DNA instead of thymidine $(T)$ during cell proliferation. The fluorescent dye Apollo ${ }^{\circledR}$ binds specifically to EdU which has inserted into DNA and could be detected by excitation light at $488 \mathrm{~nm}$. Green light represents the growing cell. Cell nuclei stained with Hoechst 33258 represent the total number of cells. In EdU incorporation assay, $1 \times 10^{4}-1.5 \times 10^{4}$ cells were seeded into 96-well plates 24 or $48 \mathrm{~h}$ after transfection and the next steps were following the manufacturer's protocol of EdU Imaging Kit (Ribobio, China), the fluorencent images were acquired by the fluorescence microscope (Olympus, Japan). All the experiments were performed for at least three times.

\section{Cell apoptosis analysis by flow cytometry}

Following the manufacturer's protocol of the Annexin V-FITC/PI Dual Staining Cell Apoptosis Detection Kit (Bestbio, China), transfected cells were plated in 12-well plates for 36 hours, and gathered together by centrifugation after washing with ice-cold PBS. Then the cells were resuspended in Annexin V binding buffer and incubated with FITC-conjugated Annexin $V$ antibody and propidium iodide (1:100 dilutions) for 15 min at room temperature. The fluorescence was detected by Beckman CytoFLEX FCM (USA).

\section{Western blotting analysis}

Cells were harvested and boiled in lysis buffer containing protease inhibitors. Total protein lysates were separated by $10 \%$ SDS-PAGE gel and electrophoretically transferred PVDF (polyvinylidene difluoride) membranes (Millipore). Then the PVDF membranes were blocked by $5 \%$ skim milk for 3-4 hours at room temperature and incubated with primary antibodies $(1: 1000)$ at $4{ }^{\circ} \mathrm{C}$ overnight, washed with TBST buffer, and incubated again with an appropriate HRP-conjugated secondary antibody $(1: 5,000)$ at room temperature for $1 \mathrm{~h}$. The enhanced chemiluminecence $(\mathrm{ECL})$ was used to detect the results.

\section{Bioinformatics analysis}

In this study, an integrating bioinformatics analysis was performed to analyze the function and interaction between LncRNA HOTAIR and miR-214-3p. The starbase v2.0 (http://starbase.sysu.edu.cn/) was used to predict the binding sites of HOTAIR and miR-214-3p. In addition, the targeted genes of miR214-3p were predicted using TargetScan7. 2(http://www.targetscan.org/) and miRWALK3.0 (http://mirwalk.umm.uni-heidelberg.de/)[34]. Subsequently, Gene Ontology (GO) and Kyoto Encyclopedia of Genes and Genomes (KEGG) pathway enrichment analysis were performed using the Search Tool for 
the Retrieval of Interacting Genes (STRING 11.0; https://string-db.org/) database, and FDR $<0.05$ was set as the cut-off criterion.

\section{Statistical analysis}

All statistical analyses were performed using SPSS 25 (SPSS Inc., Chicago, IL, USA). The results were expressed as the means \pm S.D. The statistical significance of the results was analyzed using two-tailed Student's $t$-test. The difference was considered statistically significant at $p<0.05$ and indicated by asterisks in the figures. All experiments were repeated independently more than three times.

\section{Results}

\section{HOTAIR expression is correlated with HPV16E7 in cervical cancer cells}

In this experiment, we detect the expression level of HPV16E7 and HOTAIR in End1/E6E7, SiHa and C-33A cells. End1/E6E7 cell lines was immortalized cervical epithelial cell where HPV16 E6 and E7 has been stably transfected, SiHa cell line has integrated with HPV16 genome, and HPV negative C-33A cells was used as negative control to normalize in this study. The results showed that comparing to C-33A cells, HOTAIR appeared to be at a high expression level in End1/E6E7 and SiHa cells, accompany by the high expression of HPV16 E7 (Fig. 1A,1B). Our results were consistent with the previous studies. Next, we detected the expression level of HOTAIR when HPV16 E7 expression was knocked down or up-expressed in those three cell lines, respectively. Our results showed that after we knocked down HPV16 E7, HOTAIR expression showed a significant decrease in End1/E6E7 and SiHa cells, but there was no obvious change in C33A cells without E7 expression at all (Fig. 1C). And while we exogenously up-expressed HPV16 E7 by transfected with pLXSN-E7, HOTAIR expression in those three cell lines were all upregulated (Fig. 1D). Our results confirmed that HOTAIR expression have positive correlation with HPV16 E7, suggested that there was indeed interaction between IncRNA HOTAIR and HPV16 E7 in cervical cancer cells.

\section{HOTAIR plays a growth promoting role in cervical cancer cells.}

To investigate the effect of HOTAIR on the phenotype of cervical cancer cells, the CCK8 and EdU incorporation assays were performed to detect the cell viability and cell proliferation, respectively. In this study, we detected cell viability at $24 \mathrm{~h}, 48 \mathrm{~h}$ and $72 \mathrm{~h}$ after HOTAIR siRNA transfection. As shown by CCK 8 assay, the cell viability was significantly inhibited in End1/E6E7 and SiHa cells at 48h and 72h (Fig. 2A). Additionally, knockdown of HOTAIR also significantly inhibited the proliferative abilities of End1/E6E7 and $\mathrm{SiHa}$ cells after HOTAIR siRNA transfection 48h (Fig. 2B). No significant changes were found in C$33 \mathrm{~A}$ cells by CCK8 and EdU incorporation assay, it was HOTAIR remained a low expression level in C-33A cells. We used FITC/PI double staining assay to detect cell apoptosis by flow cytometry. Results showed 
that the apoptotic rate significantly increased after knocking down HOTAIR expression in $\mathrm{SiHa}$ and End1/E6E7 cells, but the apoptotic rate of C-33A cells remained almost unchanged (Fig. 2C). By contrast, for the HPV negative cells, the proliferation of C-33A showed an obvious increase (Fig. 2D), and the apoptotic rate of C-33A cells showed a significant decrease when HOTAIR functional motif was overexpressed by transfected with pEGFP-C1-HOTAIR-A in C-33A cells (Fig. 2E). Taken together, these results suggested that HOTAIR could promote proliferation and inhibit apoptosis in HPV16 positive cervical cancer cells.

\section{miR-214-3p is downregulated expression and acts as a suppressor in HPV16 positive cervical cancer cells}

As shown in Fig. 3A, the expression miR-214-3p was downregulated in End1/E6E7 and SiHa cells comparing to HPV negative C-33A cells. We also investigated the effect of miR-214-3p on cell proliferation and apoptosis in three cell lines. Results revealed that overexpression of miR-214-3p could inhibit cell proliferation of End1/E6E7 and SiHa cells (Fig. 3B\&C). We also observed that End1/E6E7 and $\mathrm{SiHa}$ cells exhibited significant difference in percentage of early apoptosis while miR-214-3p overexpressed. However, in case of C33A cells there is no significant difference percentage of early apoptotic cells in miR-214-3p overexpressed cells as compared to control cells. Notability, these results were completely contrary to the function and effect of HOTAIR in cervical cancer cells.

\section{HOTAIR serves as a sponge for miR-214-3p}

To clarify whether there was an interaction between HOTAIR and miR-214-3p, we performed an integrating bioinformatics analysis to identify the potential binding site on two non-coding RNAs with starbase v2.0 database. Finally, we found that the a $7 \mathrm{bp}$ seed sequences (CAGCAGG) at 5 ' end of miR214-3p were fully complementary to the 1807-1813 bp on HOTAIR (Fig. 4A). We performed dual luciferase assay to verify the function of this binding site as a microRNA response elements (MREs)on HOTAIR. First, we obtained a $600 \mathrm{bp}$ fragments of the predicted binding site flank sequence (including the upstream $300 \mathrm{bp}$ and downstream $300 \mathrm{bp}$ ) by PCR. Then, this $600 \mathrm{bp}$ fragment was inserted into a reporter plasmid which expresses luciferase, the Renilla luciferase plasmid was used as a control. In order to avoid the influence of abnormal oncogene expression patterns and HPV interference in tumor cells, we performed the dual luciferase assay in HEK-293T cells. The results shown that the activity of luciferase was significantly reduced after co-transfected with miR-214-3p mimics and HOTAIR reporter plasmid, indicating that this site was indeed a response element on HOTAIR sequence (Fig. 4B). HOTAIR could rely on this response element to serve as a sponge against miR-214-3p, and inhibited the function of miR-214$3 p$ in cervical cancer cells.

Next, we focused our analysis on the expression of miR-214-3p in cells transfected with HOTAIR siRNA. Results suggested that the expression of miR-214-3p was significantly increased after HOTAIR knocked down in HPV16 positive End1/E6E7 and SiHa cells. However, in HPV negative C-33A cells, knockdown of HOTAIR did not significantly change the expression of miR-214-3p (Fig. 4C). As shown in Fig. 4D, it was 
found that miR-214-3p expression was obviously inhibited when HOTAIR-A plasmid which contained the binding sites of miR-214-3p was transfected into C-33A cells.

To verify the correlation between HPV16 E7 and HOTAIR expression, we detected the alteration of miR214-3p expression when knocked down and overexpressed HPV16 E7, respectively. Results indicated that miR-214-3p expression could be significantly upregulated by transfected with sh-E7 in End1/E6E7 and $\mathrm{SiHa}$ cells (Fig. 4E). Conversely, the expression of miR-214-3p was significantly downregulated when HPV16E7 was upregulated in these cells by transfected with E7 expression plasmid into cells (Fig. 4F). Considering all the results above, it suggested that HOTAIR could act as a molecular sponge of miR-214$3 p$, to inhibit the action of miR-214-3p in HPV16 positive End1/E6E7 and SiHa cells. Notably, HPV16 E7 maybe play a key regulatory role in this process, because the inhibition roles of HOTAIR against miR-214$3 p$ no longer existed due to the loss or decline of E7 expression in cervical cancer cells.

\section{HOTAIR controls $\beta$-catenin expression via miR-214-3p}

To explore the potential signaling pathway regulated by miR-214-3p in cervical cancer cells, we performed the bioinformatics analysis of miR-214-3p. Firstly, target genes of miR-214-3p were predicted with TargetScan7.2 and miRWALK3.0. After discarding repeatedly predicted genes, the predicted target genes were further analyzed by performing a negative correlation analysis. Finally, a total of 1451 genes were predicted as target genes of miR-214-3p (Fig. 5A). Next, to initially comprehend the action of the genes, we submitted predicted genes to STRING11.0, to perform the Gene ontology (GO) and KEGG Pathway enrichment analysis. GO enrichment analysis results showed that mostly enriched in biological processes (BP) terms were related to cellular process, regulation of biological process, reactome pathways (RCTM) terms included Wnt ligand biogenesis and trafficking, nuclear Events (kinase and transcription factor activation), and MAPK targets/Nuclear events mediated by MAP kinases9 (Table 2). Pathway enrichment analysis results showed that the predicted target genes were significantly enriched in Wnt signaling pathway, hippo signaling pathway, signaling pathways regulating pluripotency of stem cells, and pathways in cancer (Fig. 5B). Taken together, these results suggested that Wnt signaling pathway was probably key pathway related to miR-214-3p.

In Wnt signaling pathway, $\beta$-catenin has been identified as a verified target of miR-214-3p by bioinformatics analysis. In this part we aimed to investigate the effect of HOTAIR/miR-214-3p on $\beta$ catenin. At first, we transfected miR-214-3p mimics into cervical cancer cells, mRNA and protein expression of $\beta$-catenin were both declined (Fig. 6A\&B), indicated that $\beta$-catenin indeed was a direct target gene of miR-214-3p. When HOTAIR was knocked down in End1/E6E7 and SiHa, $\beta$-catenin mRNA and protein expression level were also significantly inhibited (Fig. 6C\&D). But the expression level of $\beta$-catenin could be up-regulated again when HOTAIR-A plasmid contained the binding sites of miR-214-3p was transfected into C-33A cells (Fig. 6E\&F). Combining with the previous results, HOTAIR and miR-214-3p could both directly regulated the expression of $\beta$-catenin, and HOTAIR could upregulate the expression of $\beta$-catenin by targeted adsorbing miR-214-3p in cervical cancer cells. In another word, HOTAIR and $\beta$ catenin could act as a pair of competitive endogenous RNA in HPV16 positive cervical cancer through 
competing binding to miR-214-3p. Finally, Wnt/ $\beta$-catenin signaling pathway might be activated by HOTAIR/ miR-214-3p axis in cervical cancer cells.

\section{Discussion}

Increasing evidence has revealed that specific IncRNAs might give a significant contribution in the progression, metastasis and chemoresistance of cancer[35]. These multiple functional tumor-associated IncRNAs have either oncogenic or tumor-suppressive properties in cancer [36]. In particular, accumulating research data have demonstrated that the IncRNAs alterations in HPV infected cells was also crucial for the progression of cervical cancer. For example, a microarray analysis revealed that thousands of host IncRNAs had differential expression in oncogenic HPV-positive cells compared to the HPV-negative cervical cancer cell line[37]. Lots of IncRNAs were found to be differentially expressed in the HPV18 positive HeLa cells respect to C33A cell line[38]. In addition, accumulating research data have demonstrated the high-risk HPV E6 and E7 oncoproteins could alter the expression of these IncRNAs and their downstream miRNAs or targets.

In general, HOTAIR has been shown to recruits chromatin-modifying proteins and to affect cancer epigenome modulation[39]. In cervical cancer, limited data suggested that HOTAIR could act as sponge for several miRNAs and cause deregulation of the respective target genes. However, the exact mechanism remains unclear. Furthermore, HOTAIR levels have shown to be strictly controlled by the HPV E7 protein in cervical cancer [22]. In this study, our results revealed that HOTAIR and miR-214-3p were both differentially expressed in HPV16 positive cervical cancer cells, HOTAIR showed significantly higher expression level, while miR-214-3p remains a lower expression level in cervical cancer cells. However, they showed exactly reverse expression tendency. Meanwhile, datasets mining also found the higher expression of HOTAIR in cancer tissues[]. These findings were consistent with previous studies[23, 24].

For better investigate the function of HOTAIR and miR-214-3p in HPV16 positive cervical cancer, we knocked down HOTAIR and overexpressed miR-214-3p in HPV16 positive cells, respectively. Our results indicated that no matter knocking down of HOTAIR or overexpressing miR-214-3p, cell proliferate ability was significantly inhibited accompanied by cellular apoptosis increased. That represents HOTAIR played a promoting role in HPV16 cervical carcinogenesis and the function of miR-214-3p was totally reverse. We supposed that HOTAIR was responsible for miR-214-3p down-regulation in HPV16 positive cervical cancer by serving as a competitive endogenous RNA through sponging mature miR-214-3p in cells. Based on bioinformatics analysis, we identified a potential binding site on HOTAIR transcript against miR-214$3 p$. Eventually A seven bp seed sequences (CAGCAGG) at 5 ' end of miR-214-3p were fully complementary to the 1807-1813 bp on HOTAIR. Moreover, the function of this binding site as a microRNA response elements (MREs)on HOTAIR was also verified by dual luciferase assay. To further confirm the interaction between HOTAIR and miR-214-3p, we detected the change of miR-214-3p expression when HOTAIR expression was interfered in cancer cells. As shown in the results, miR-214-3p expression decreased accompany with the up-regulation of HOTAIR. Our results revealed a strong negative correlation between HOTAIR and miR-214-3p expression. 
In above results, we had confirmed that HPV16E7 could upgrade HOTAIR expression. Next, when down or up-regulated expression of HPV16 E7 respectively, the expression of HOTAIR showed the same trend. In addition, miR-214-3p expression was significantly increased when HPV16 E7 expression was knocked down, but once HPV16 E7 gene was up-regulated expression again in cancer cells, HOTAIR expression went up also and the expression trend of miR-214-3p was obviously reversed. The results suggest that the infection status of HPV16 played an important role in regulating expression of HOTAIR or miR-214-3p in cervical cancer cells. Consequently, we speculated that the main cause of the above phenomenon was attributed to losing the sponge effect of HOTAIR against miR-214-3p.

Based on bioinformatics analysis, Wnt/ $\beta$-catenin signaling pathway was one of the key pathways implicated in miR-214-3p regulation network in cervical cancer. Interestingly, Wnt/ $\beta$-catenin signaling pathway contains some key genes, such as CTNNB1( $\beta$-catenin), PLCB4, PSEN1, PPP2CB, were all the well-recognized target gene for miR-214-3p by bioinformatics analysis. The studies found that miR-214$3 p$ could degrade or inactivate the $\beta$-catenin gene by binding to the 3 'UTR of $\beta$-catenin mRNA in various malignant tumors including breast cancer and esophageal cancer $[40,41]$. In our study, $\beta$-catenin was significantly decreased at both mRNA and protein level when miR-214-3p was up expressed in HPV16 cervical cancer cells. And once HOTAIR was knocked down in HPV16 positive cervical cancer cells, miR214-3p could escape from the absorption of HOTAIR and further lead to its target gene $\beta$-catenin been degraded. HOTAIR in HPV-negative C-33A cells remains an extremely lower level, so when we overexpressed the HOTAIR functional region in C-33A cells, the free miR-214-3p lost its inhibitory effect against $\beta$-catenin mRNA due to the adsorption of HOTAIR against miR-214-3p, eventually caused the expression of $\beta$-catenin was significantly up-regulated in cervical cancer cells.

Wnt/ $\beta$-catenin dependent pathway also known as the canonical pathway. When Wnt/ $\beta$-catenin dependent pathway was activated, $\beta$-catenin protein was accumulated in the cytoplasm, then it was transferred to the nucleu and interacted with T cell factor (TCF)/Lymphoid Enhancer Factor (LEF) transcription factors, and promotes the transcription of downstream targets, such as cyclin D1, c-Myc, and matrix metalloproteinase 1[42]. On this basis, Wnt/ $\beta$-catenin signaling pathway played modulation roles in cancer cells apoptosis, proliferation, invasion, and migration in the progression of various cancers[43]. In this study, we found when HOTAIR was knocked down in HPV16 positive cervical cancer cells, the expression of $\beta$-catenin was significantly inhibited. We supposed that the lower expression of HOTAIR lost the sponge effect against miR-214-3p, further lead to degradation of $\beta$-catenin mRNA, then wnt/ $\beta$ catenin signaling pathway was inhibited in cells. Oppositely, in HPV16-positive cervical cancer cells, HOTAIR was highly expressed due to the presence of HPV16E7, then miR-214-3p was adsorbed and loses its effect on target genes, and $\beta$-catenin expression was released, resulting in malignant phenotype of cervical cancer cells. In summary, HOTAIR and miR-214-3p might form a regulated axis and play important regulation roles in the malignant behaviour of HPV16-positive cervical cancer cells. Also, canonical wnt/ $\beta$-catenin signaling pathway was a key part of this ceRNA regulatory network in cervical cancer. 
At present, cervical cancer continues to represent an important health problem for in women worldwide. Although the epigenetic alterations in HPV infected cells indeed be crucial for the progression to cervical cancer, studies on the correlation between HPV and intracellular ncRNA were very limited so far. Meanwhile, the different investigations provided conflicting results with a significant proportion of ncRNAs being upregulated in one study but downregulated in another study. Therefore, characterization of the complex relationship between ncRNAs and target genes were needed to further carried out, help to improve the early diagnosis and treatment of cervical cancer.

\section{Conclusion}

In conclusion, we found a novel regulatory axis: HOTAIR/miR-214-3p/ $\beta$-catenin, exerts a ceRNA effect, involved in HPV16 positive cervical cancer cells proliferation and apoptosis. Our results provided some potential therapeutic targets and prognostic indicators of cervical cancer.

\section{Declarations}

\section{Acknowledgments}

Not applicable

\section{Authors' contributions}

ZM conceived and designed the experiments; ZY and WYQ performed experiments; LMY and WDQ analyzed the data; $Z Y$ and WYQ wrote the manuscript. All authors read and approved the fial manuscript.

\section{Ethics approval and consent to participate}

Not applicable.

\section{Consent for publication}

Not applicable.

\section{Competing interests}

The authors declare that they have no competing interests

\section{Funding}

Not applicable

\section{References}


1. Erratum. Global cancer statistics 2018: GLOBOCAN estimates of incidence and mortality worldwide for 36 cancers in 185 countries. CA Cancer J Clin. 2020;70:313.

2. zur Hausen H. Papillomaviruses in the causation of human cancers - a brief historical account. Virology. 2009;384:260-5.

3. Tornesello ML, Annunziata C, Tornesello AL, Buonaguro L, Buonaguro FM. Human Oncoviruses and p53 Tumor Suppressor Pathway Deregulation at the Origin of Human Cancers. Cancers (Basel) 10, 2018.

4. Yeo-Teh NSL, Ito Y, Jha S. High-Risk Human Papillomaviral Oncogenes E6 and E7 Target Key Cellular Pathways to Achieve Oncogenesis. Int J Mol Sci 19, 2018.

5. Chhabra R. Cervical cancer stem cells: opportunities and challenges. J Cancer Res Clin Oncol. 2015;141:1889-97.

6. Dueñas-González A, Campbell S. Global strategies for the treatment of early-stage and advanced cervical cancer. Curr Opin Obstet Gynecol. 2016;28:11-7.

7. Nicoli F, Mantelli B, Gallerani E, et al.: HPV-Specific Systemic Antibody Responses and Memory B Cells are Independently Maintained up to 6 Years and in a Vaccine-Specific Manner Following Immunization with Cervarix and Gardasil in Adolescent and Young Adult Women in Vaccination Programs in Italy. Vaccines 8, 2020.

8. Hong S, Cheng S, Songock W, Bodily J, Laimins LA. Suppression of MicroRNA 424 Levels by Human Papillomaviruses Is Necessary for Differentiation-Dependent Genome Amplification. J Virol 91, 2017.

9. He Z, Xu Z, Hang D, et al. Anti-HPV-E7 seropositivity and risk of esophageal squamous cell carcinoma in a high-risk population in China. Carcinogenesis. 2014;35:816-21.

10. Roberts TC, Morris KV, Weinberg MS. Perspectives on the mechanism of transcriptional regulation by long non-coding RNAs. Epigenetics. 2014;9:13-20.

11. Sun Q, Hao Q, Prasanth KV. Nuclear Long Noncoding RNAs: Key Regulators of Gene Expression. Trends Genet. 2018;34:142-57.

12. Dong J, Su M, Chang W, Zhang K, Wu S, Xu T. Long non-coding RNAs on the stage of cervical cancer (Review). Oncol Rep. 2017;38:1923-31.

13. Sharma S, Munger K. Expression of the cervical carcinoma expressed PCNA regulatory (CCEPR) long noncoding RNA is driven by the human papillomavirus E6 protein and modulates cell proliferation independent of PCNA. Virology. 2018;518:8-13.

14. He H, Liu X, Liu Y, et al.: Human Papillomavirus E6/E7 and Long Noncoding RNA TMPOP2 Mutually Upregulated Gene Expression in Cervical Cancer Cells. J Virol 93, 2019.

15. Chen F-J, Sun M, Li S-Q, et al. Upregulation of the long non-coding RNA HOTAIR promotes esophageal squamous cell carcinoma metastasis and poor prognosis. Mol Carcinog. 2013;52:90815.

16. Zhang J-X, Han L, Bao Z-S, et al. HOTAIR, a cell cycle-associated long noncoding RNA and a strong predictor of survival, is preferentially expressed in classical and mesenchymal glioma. Neuro Oncol. 
2013;15:1595-603.

17. Zhang Y, Ai H, Fan X, Chen S, Wang Y, Liu L. Knockdown of long non-coding RNA HOTAIR reverses cisplatin resistance of ovarian cancer cells through inhibiting miR-138-5p-regulated EZH2 and SIRT1. Biol Res. 2020;53:18.

18. Zhao W, Song M, Zhang J, Kuerban M, Wang H. Combined identification of long non-coding RNA CCAT1 and HOTAIR in serum as an effective screening for colorectal carcinoma. Int J Clin Exp Pathol. 2015;8:14131-40.

19. Yang S-Z, Xu F, Zhou T, Zhao X, McDonald JM, Chen Y. The long non-coding RNA HOTAIR enhances pancreatic cancer resistance to TNF-related apoptosis-inducing ligand. J Biol Chem.

2017;292:10390-7.

20. Kim HJ, Lee DW, Yim GW, Nam EJ, Kim S, Kim SW, Kim YT. Long non-coding RNA HOTAIR is associated with human cervical cancer progression. Int J Oncol. 2015;46:521-30.

21. Huang L, Liao L-M, Liu A-W, Wu J-B, Cheng X-L, Lin J-X, Zheng M. Overexpression of long noncoding RNA HOTAIR predicts a poor prognosis in patients with cervical cancer. Arch Gynecol Obstet. 2014;290:717-23.

22. Sharma S, Mandal P, Sadhukhan T, et al. Bridging Links between Long Noncoding RNA HOTAIR and HPV Oncoprotein E7 in Cervical Cancer Pathogenesis. Sci Rep. 2015;5:11724.

23. Liu M, Jia J, Wang X, Liu Y, Wang C, Fan R. Long non-coding RNA HOTAIR promotes cervical cancer progression through regulating BCL2 via targeting miR-143-3p. Cancer Biol Ther. 2018;19:391-9.

24. Li Q, Feng Y, Chao X, et al.: HOTAIR contributes to cell proliferation and metastasis of cervical cancer via targetting miR-23b/MAPK1 axis. Biosci Rep 38, 2018.

25. Pardini B, De Maria D, Francavilla A, Di Gaetano C, Ronco G, Naccarati A. MicroRNAs as markers of progression in cervical cancer: a systematic review. BMC Cancer. 2018;18:696.

26. He Y, Lin J, Ding Y, et al. A systematic study on dysregulated microRNAs in cervical cancer development. Int J cancer. 2016;138:1312-27.

27. Tornesello ML, Faraonio R, Buonaguro L, et al. The Role of microRNAs, Long Non-coding RNAs, and Circular RNAs in Cervical Cancer. Front Oncol. 2020;10:150.

28. Lui W-O, Pourmand N, Patterson BK, Fire A. Patterns of known and novel small RNAs in human cervical cancer. Cancer Res. 2007;67:6031-43.

29. Nahand JS, Taghizadeh-Boroujeni S, Karimzadeh M, et al. microRNAs: New prognostic, diagnostic, and therapeutic biomarkers in cervical cancer. J Cell Physiol. 2019;234:17064-99.

30. Romano G, Veneziano D, Acunzo M, Croce CM. Small non-coding RNA and cancer. Carcinogenesis. 2017;38:485-91.

31. Tang Q, Zheng F, Liu Z, et al. Novel reciprocal interaction of IncRNA HOTAIR and miR-214-3p contribute to the solamargine-inhibited PDPK1 gene expression in human lung cancer. J Cell Mol Med. 2019;23:7749-61. 
32. Sen P, Ghosal S, Hazra R, Mohanty R, Arega S, Sahu B, Ganguly N. CRISPR-mediated knockdown of miR-214 modulates cell fate in response to anti-cancer drugs in HPV-negative and HPVpositive cervical cancer cells. J Biosci 45, 2020.

33. Wang J-M, Ju B-H, Pan C-J, et al. MiR-214 inhibits cell migration, invasion and promotes the drug sensitivity in human cervical cancer by targeting FOXM1. Am J TransI Res. 2017;9:3541-57.

34. Sticht C, De La Torre C, Parveen A, Gretz N. miRWalk: An online resource for prediction of microRNA binding sites. PLoS One. 2018;13:e0206239.

35. Diamantopoulos MA, Tsiakanikas P, Scorilas A. Non-coding RNAs: the riddle of the transcriptome and their perspectives in cancer. Ann Transl Med. 2018;6:241.

36. Yang Z, Li X, Yang Y, He Z, Qu X, Zhang Y. Long noncoding RNAs in the progression, metastasis, and prognosis of osteosarcoma. Cell Death Dis. 2016;7:e2389.

37. Casarotto M, Fanetti G, Guerrieri R, et al.: Beyond MicroRNAs: Emerging Role of Other Non-Coding RNAs in HPV-Driven Cancers. Cancers (Basel) 12, 2020.

38. Yang L, Yi K, Wang H, Zhao Y, Xi M. Comprehensive analysis of IncRNAs microarray profile and mRNA-IncRNA co-expression in oncogenic HPV-positive cervical cancer cell lines. Oncotarget. 2016;7:49917-29.

39. Ren Y, Wang Y-F, Zhang J, Wang Q-X, Han L, Mei M, Kang C-S. Targeted design and identification of AC1NOD4Q to block activity of HOTAIR by abrogating the scaffold interaction with EZH2. Clin Epigenetics. 2019;11:29.

40. Xu Y, Lu S. Regulation of $\beta$-catenin-mediated esophageal cancer growth and invasion by miR-214. Am J Transl Res. 2015;7:2316-25.

41. Yi S-J, Li L-L, Tu W-B. MiR-214 negatively regulates proliferation and WNT/ $\beta$-catenin signaling in breast cancer. Eur Rev Med Pharmacol Sci. 2016;20:5148-54.

42. Krishnamurthy N, Kurzrock R. Targeting the Wnt/beta-catenin pathway in cancer: Update on effectors and inhibitors. Cancer Treat Rev. 2018;62:50-60.

43. Lee M, Kim HJ, Kim SW, et al. The long non-coding RNA HOTAIR increases tumour growth and invasion in cervical cancer by targeting the Notch pathway. Oncotarget. 2016;7:44558-71.

\section{Tables}


Table 1

Sequences of Primers

\begin{tabular}{|ll|}
\hline Primers & Sequences \\
\hline miR-214-RT & 5'-GTCGTATCCAGTGCAGGGTCCGAGGTATTCGCACTGGATACGACACTGCC - 3' \\
U6-RT & 5'-GTCGTATCCAGTGCAGGGTCCGAGGTATTCGCACTGGATACGACAAAATATG-3' \\
\hline miR-214-3p-real-F & 5'- CGCCGACAGCAGGCACA - 3' \\
\hline U6- real-F & 5'-GCGCGTCGTGAAGCGTTC-3' \\
miR-real-GP & 5'-GTGCAGGGTCCGAGGT-3' \\
HOTAIR-real-F & 5'-GGGACAGAAGGAAAGCCCTC-3' \\
HOTAIR-real-R & 5'-TTGAGAGCACCTCCGGGATA-3' \\
GAPDH-real-F & 5'-CTGCACCACCAACTGCTTAG-3' \\
GAPDH-real-R & 5'-TTCTGGGTGGCAGTGATG-3' \\
Luc-HOTAIR-F & 5'-CGACGCGTAGAAGCAAAGGTCCAG-3' \\
Luc-HOTAIR-R & 5'-CGCAAGCTTAAGTGCATACCTACC-3' \\
HOTAIR-A-F & 5'-CGAAGCTTAGAAGCAAAGGTCCAG-3' \\
HOTAIR-A-R & 5'-CGACGCGTAAGTGCATACCTAC-3' \\
\hline
\end{tabular}


Table 1

GO and pathway enrichment analysis of target genes of miR-214-3p

\begin{tabular}{|c|c|c|c|c|}
\hline term ID & term description & $\begin{array}{l}\text { gene } \\
\text { count }\end{array}$ & strength & FDR \\
\hline \multicolumn{5}{|l|}{$\begin{array}{l}\text { Biological } \\
\text { processes }\end{array}$} \\
\hline GO:0009987 & cellular process & 1186 & 0.03 & $\begin{array}{l}1.1 \mathrm{E}- \\
04\end{array}$ \\
\hline G0:0065007 & biological regulation & 979 & 0.04 & $\begin{array}{l}1.1 \mathrm{E}- \\
04\end{array}$ \\
\hline GO:0050789 & regulation of biological process & 942 & 0.05 & $\begin{array}{l}2.92 \mathrm{E}- \\
05\end{array}$ \\
\hline GO:0050794 & regulation of cellular process & 896 & 0.05 & $\begin{array}{l}2.88 \mathrm{E}- \\
05\end{array}$ \\
\hline GO:0008152 & metabolic process & 818 & 0.05 & $\begin{array}{l}1.1 \mathrm{E}- \\
04\end{array}$ \\
\hline \multicolumn{5}{|c|}{ cell component } \\
\hline GO:0005623 & cell & 1312 & 0.03 & $\begin{array}{l}1.39 \mathrm{E}- \\
07\end{array}$ \\
\hline GO:0005622 & intracellular & 1225 & 0.06 & $\begin{array}{l}7.97 \mathrm{E}- \\
16\end{array}$ \\
\hline G0:0043226 & organelle & 1062 & 0.05 & $\begin{array}{l}5.67 \mathrm{E}- \\
09\end{array}$ \\
\hline GO:0043229 & intracellular organelle & 1042 & 0.05 & $\begin{array}{l}1.28 \mathrm{E}- \\
08\end{array}$ \\
\hline GO:0005737 & cytoplasm & 993 & 0.07 & $\begin{array}{l}1.68 \mathrm{E}- \\
11\end{array}$ \\
\hline \multicolumn{5}{|l|}{$\begin{array}{l}\text { Molecular } \\
\text { function }\end{array}$} \\
\hline GO:0005488 & binding & 1006 & 0.05 & $\begin{array}{l}3.75 \mathrm{E}- \\
06\end{array}$ \\
\hline GO:0005515 & protein binding & 614 & 0.09 & $\begin{array}{l}1.33 \mathrm{E}- \\
06\end{array}$ \\
\hline GO:0043167 & ion binding & 541 & 0.07 & $\begin{array}{l}1.1 \mathrm{E}- \\
03\end{array}$ \\
\hline GO:0003824 & catalytic activity & 499 & 0.07 & $\begin{array}{l}2.6 \mathrm{E}- \\
03\end{array}$ \\
\hline
\end{tabular}

Abbreviations: GO, gene ontology; KEGG, Kyoto Encyclopedia of Genes and Genomes; FDR, false discovery rate. 


\begin{tabular}{|c|c|c|c|c|}
\hline term ID & term description & $\begin{array}{l}\text { gene } \\
\text { count }\end{array}$ & strength & FDR \\
\hline GO:0097159 & organic cyclic compound binding & 464 & 0.06 & $\begin{array}{l}3.41 \mathrm{E}- \\
02\end{array}$ \\
\hline \multicolumn{5}{|c|}{ KEGG pathways } \\
\hline hsa04310 & Wnt signaling pathway & 32 & 0.47 & $\begin{array}{l}1.1 \mathrm{E}- \\
04\end{array}$ \\
\hline hsa04390 & Hippo signaling pathway & 30 & 0.42 & $\begin{array}{l}7.5 \mathrm{E}- \\
04\end{array}$ \\
\hline hsa04550 & $\begin{array}{l}\text { Signaling pathways regulating pluripotency of } \\
\text { stem cells }\end{array}$ & 28 & 0.43 & $\begin{array}{l}7.5 \mathrm{E}- \\
04\end{array}$ \\
\hline hsa04261 & Adrenergic signaling in cardiomyocytes & 27 & 0.41 & $\begin{array}{l}3.7 \mathrm{E}- \\
03\end{array}$ \\
\hline hsa05200 & Pathways in cancer & 67 & 0.24 & $\begin{array}{l}3.7 \mathrm{E}- \\
03\end{array}$ \\
\hline \multicolumn{5}{|l|}{$\begin{array}{l}\text { Reactome } \\
\text { Pathways }\end{array}$} \\
\hline HSA-3238698 & WNT ligand biogenesis and trafficking & 11 & 0.75 & $\begin{array}{l}2.23 \mathrm{E}- \\
02\end{array}$ \\
\hline HSA-198725 & $\begin{array}{l}\text { Nuclear Events (kinase and transcription factor } \\
\text { activation) }\end{array}$ & 9 & 0.68 & $\begin{array}{l}4.12 \mathrm{E}- \\
02\end{array}$ \\
\hline HSA-450282 & $\begin{array}{l}\text { MAPK targets/ Nuclear events mediated by } \\
\text { MAP kinases }\end{array}$ & 10 & 0.63 & $\begin{array}{l}4.12 \mathrm{E}- \\
02\end{array}$ \\
\hline HSA-8986944 & Transcriptional Regulation by MECP2 & 16 & 0.56 & $\begin{array}{l}2.23 \mathrm{E}- \\
02\end{array}$ \\
\hline HSA-187037 & Signaling by NTRK1 (TRKA) & 18 & 0.5 & $\begin{array}{l}2.23 \mathrm{E}- \\
02\end{array}$ \\
\hline
\end{tabular}

\section{Figures}



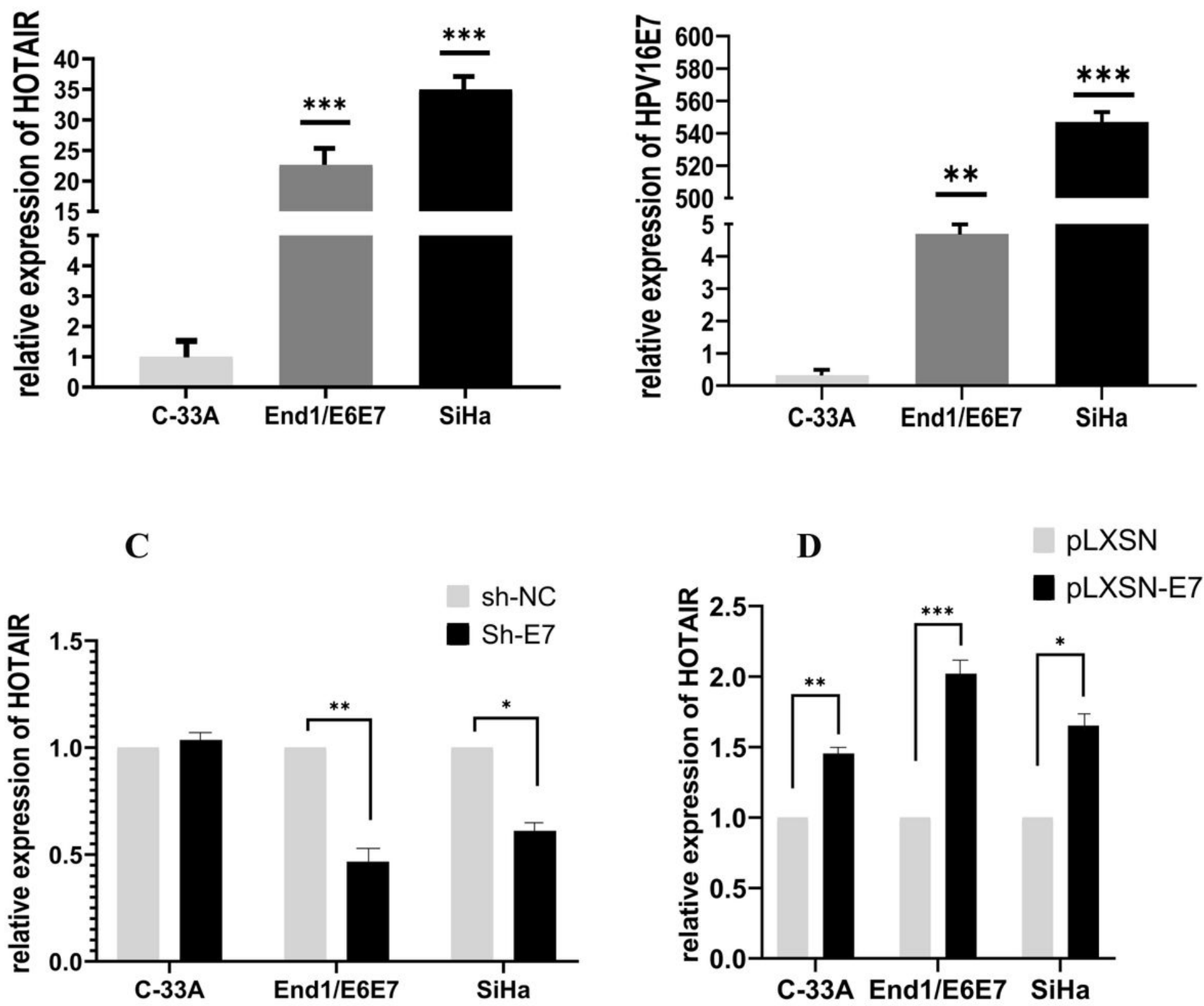

D

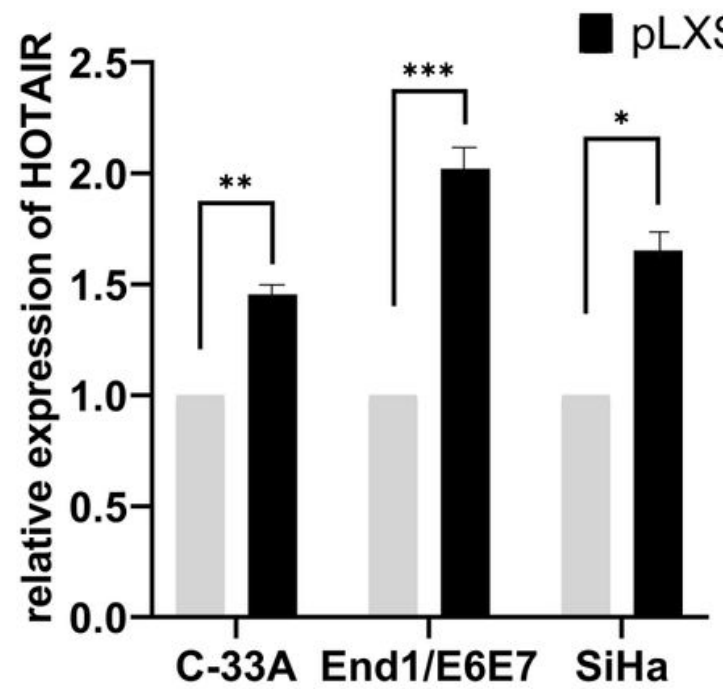

Figure 1

HPV16 E7 promotes HOTAIR expression in cervical cancer cells by qPCR analysis. (A) HOTAIR was highly expressed in End1/E6E7 and SiHa cells, while expressed very low in C-33A cells (HPV negative). (B) HPV16 E7 was highly expressed in End1/E6E7 and SiHa cells. compared with C-33A cell, **: $P<0.01$, ***: $P<0.001$. (C) Knock down HPV16 E7 significantly inhibited the expression of HOTAIR in End1/E6E7 and $\mathrm{SiHa}$ cells, but there was no alteration in C-33A cell. (D) Upregulated expression of HPV16 E7 significantly promoted HOTAIR expression in all cervical cancer cells. The mean values and standard error were obtained from three independent experiments. GAPDH was used as an endogenous control gene. *: $\mathrm{P}<$ 0.05, , $*$ P $<0.01$. 


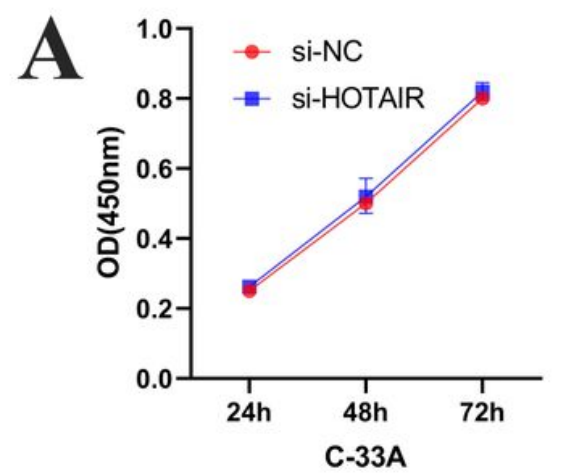

B

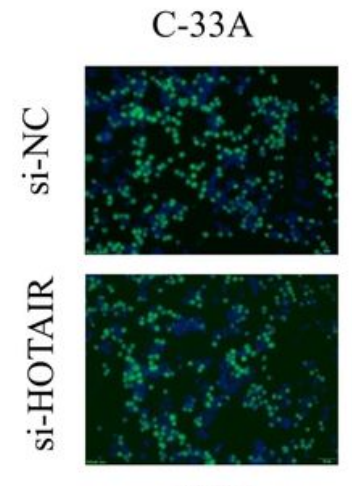

C
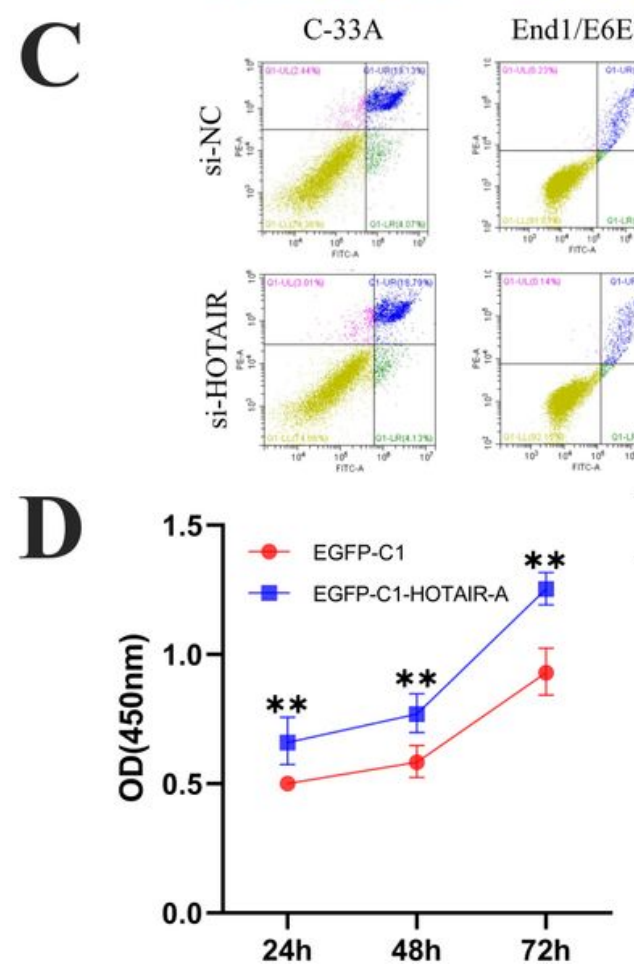
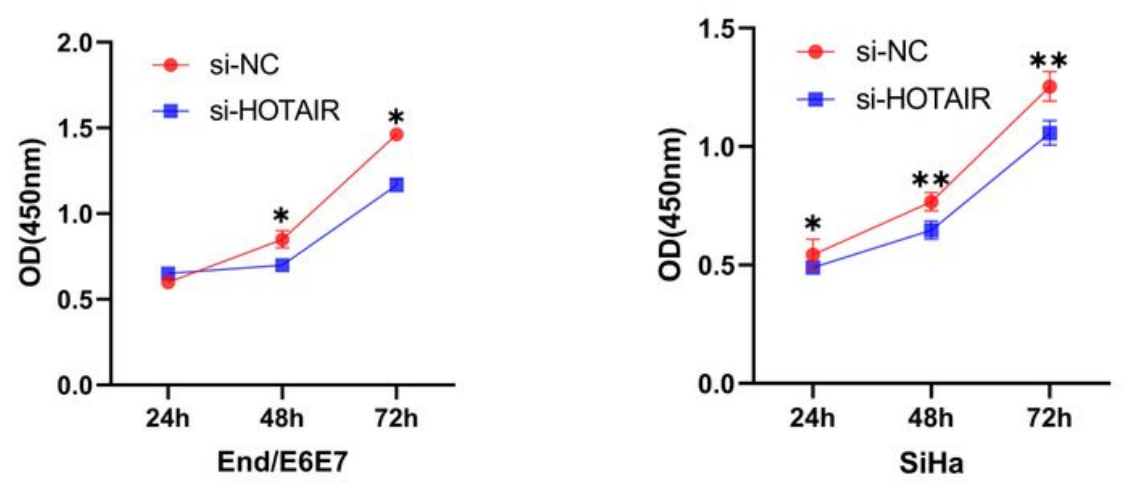

End1/E6E7
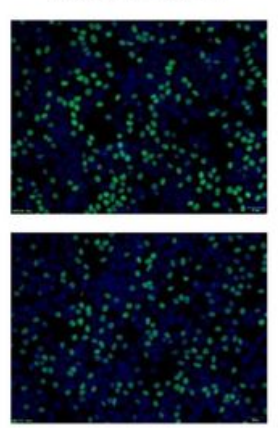

$\mathrm{SiHa}$
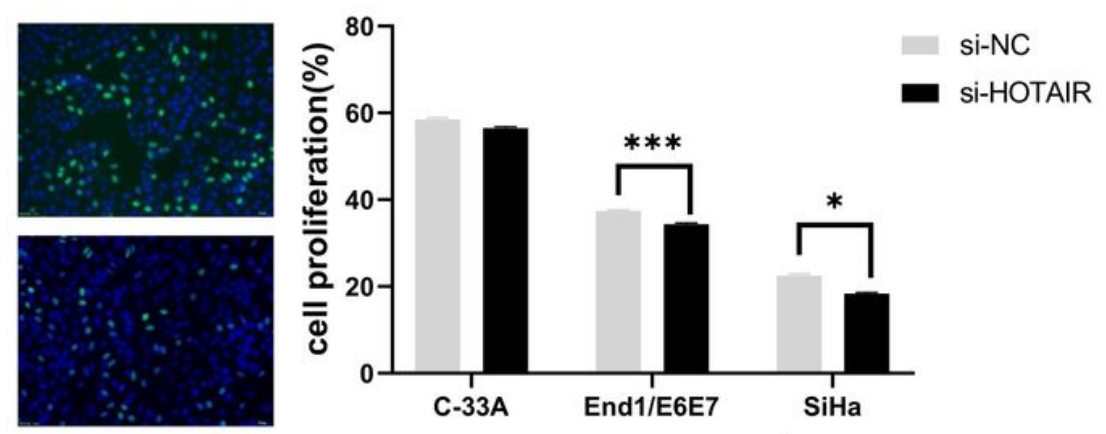

End1/E6E7
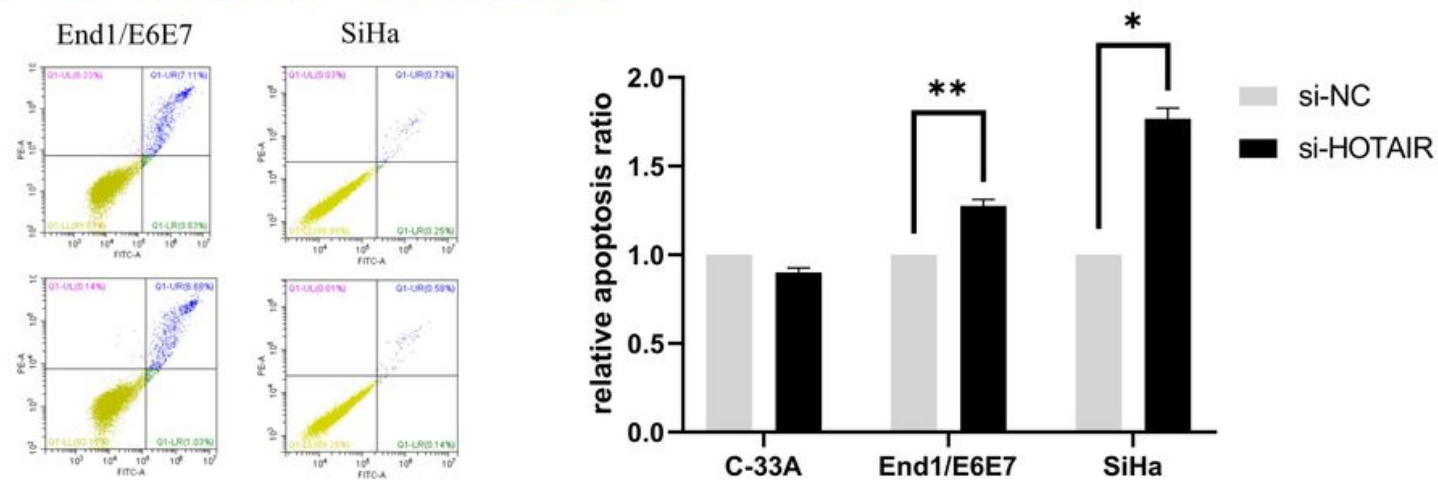

$\mathbf{E}$
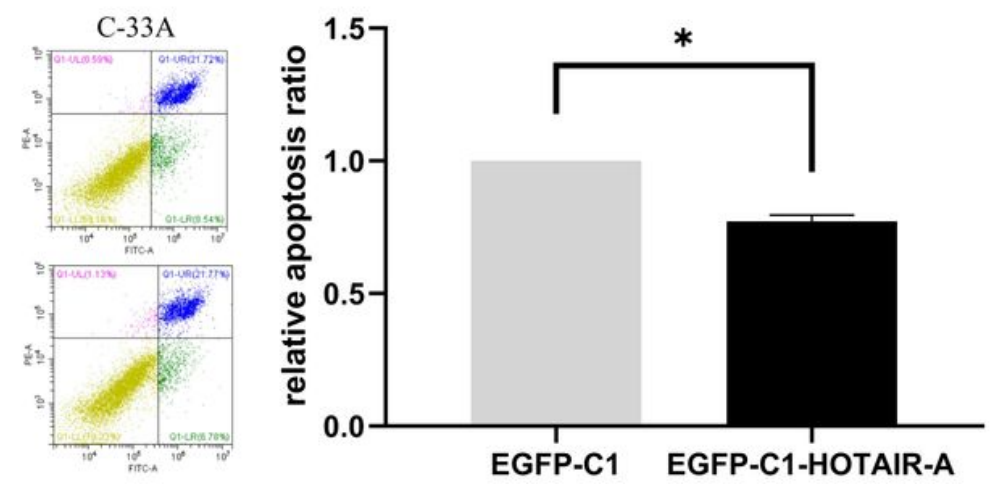

Figure 2

HOTAIR plays a growth promoting role in cervical cancer cells. (A) C-33A, End1/E6E7 and SiHa cells were transfected with HOTAIR siRNA for $24 \mathrm{~h}, 48 \mathrm{~h}$ and $72 \mathrm{~h}$, the cell viability was measured by CCK8 assay. Results showed that the cell viability of End1/E6E7 and SiHa cells were significantly inhibited, but there was no significant change in C-33A. (B) Cell proliferation measured by EdU incorporation assay after HOTAIR knocked down. The cell proliferation of End1/E6E7 and SiHa cells were significantly inhibited. (C) The cell apoptosis was analyzed by Flow cytometry analysis. HOTAIR knockdown significantly increased 
the percentage of apoptotic cells in SiHa and End1/E6E7 cells. (D) The cell viability was obvious increasing when overexpressed functional motif of HOTAIR in C-33A cells by CCK8 assay. (E) The early apoptosis of C-33A cell was significantly decreased after overexpressed HOTAIR-A by flow cytometry analysis. The mean values and standard error were obtained from three independent experiments. *: $\mathrm{P}<$ $0.05, * *: P<0.01$.

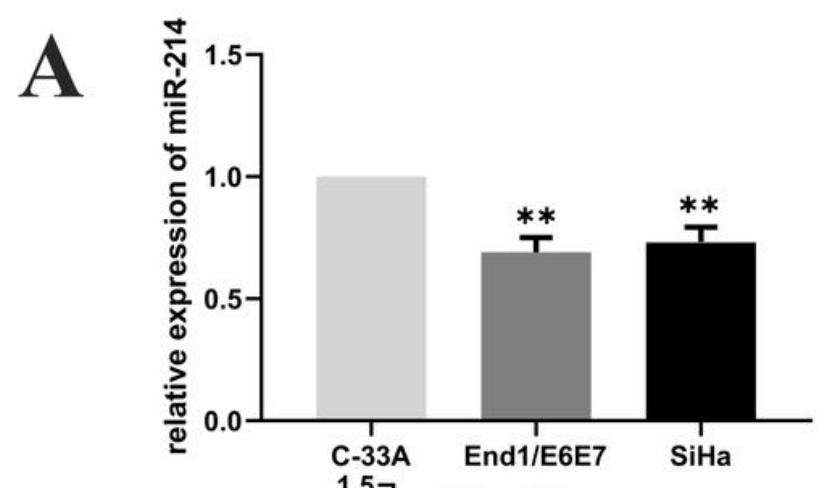

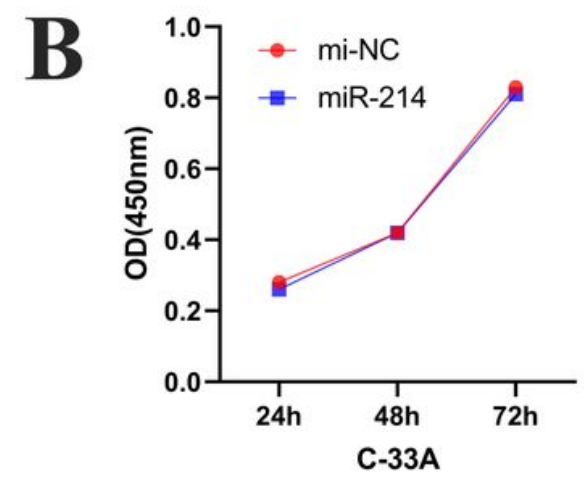

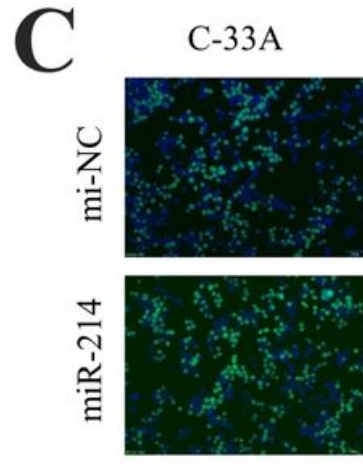

D
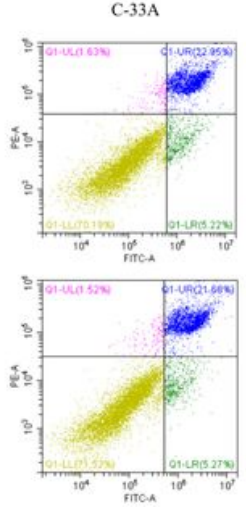

End1/E6E7
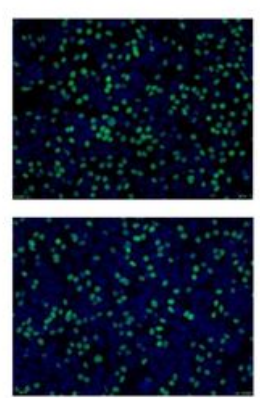

End1 /E6E7
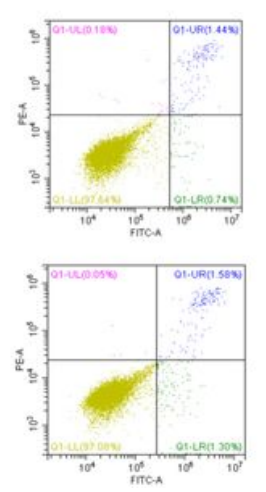
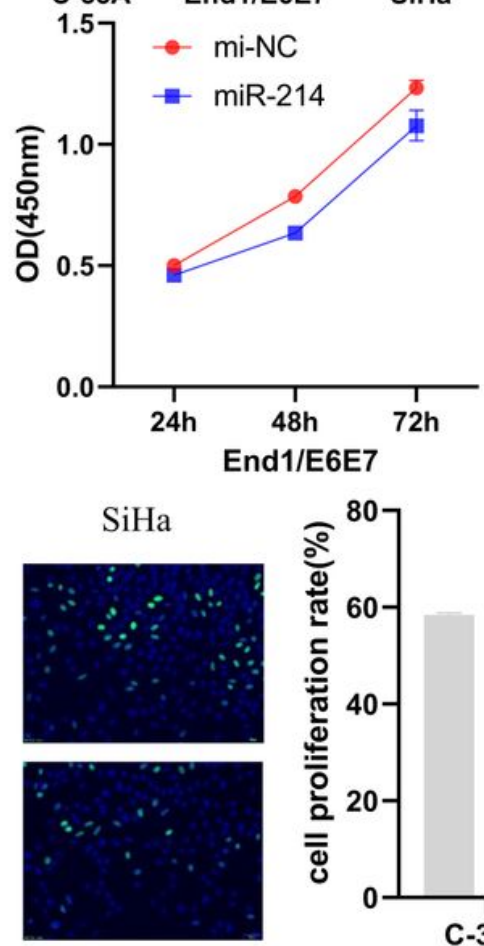

SiHa
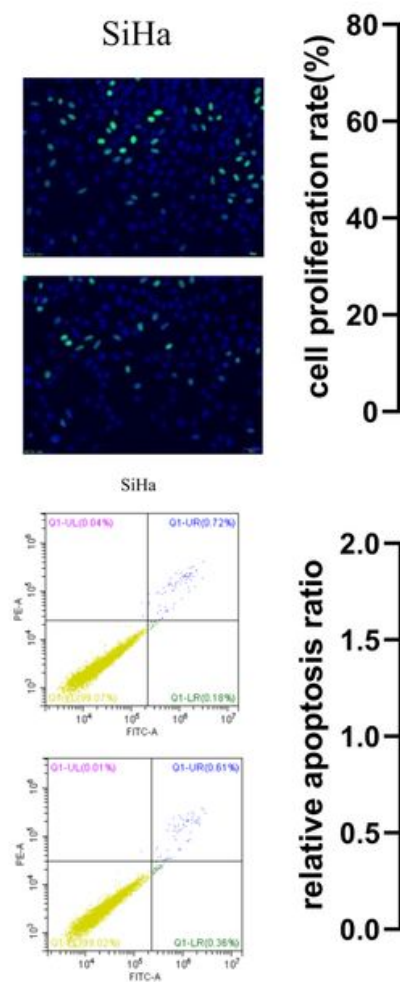

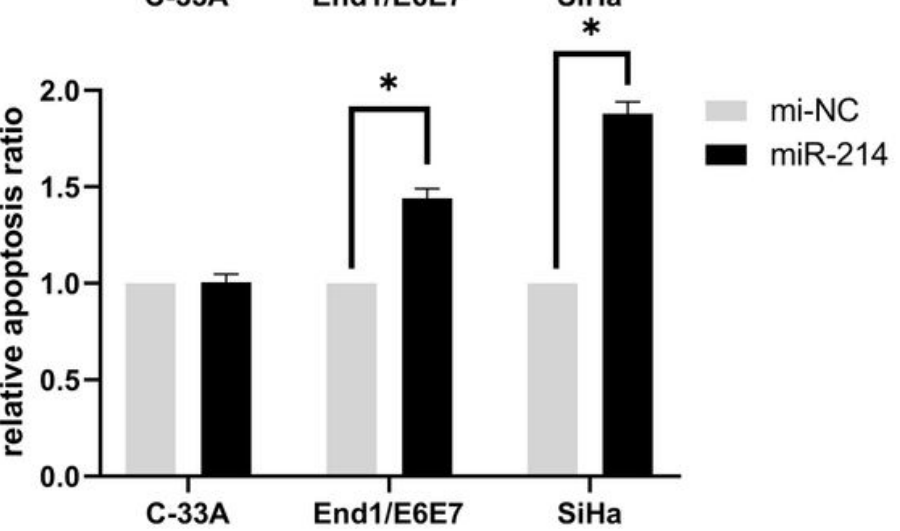

Figure 3 
miR-214-3p was down regulated expression in HPV16 positive cells and acted as a suppressor gene in cervical cancer cells. (A) The expression miR-214-3p in End1/E6E7 and SiHa cells was significantly lower than in C-33A cells. (B \& C) C-33A, End1/E6E7 and SiHa cells were transfected with miR-214-3p mimic and negative control for $24 \mathrm{~h}, 48 \mathrm{~h}$ and $72 \mathrm{~h}$, the cell viability was measured by CCK 8 assay and cell proliferation was measured by EdU incorporation assay. Results showed that the cell viability and proliferation of End1/E6E7 and SiHa cells were significantly inhibited, but there was no significant change in C-33A cells. (D) Overexpression of miR-214 increases early apoptotic population in cervical cancer cells after transfected with miR-214-3p mimic. The mean values and standard error were obtained from three independent experiments. *: $P<0.05, * \star$ : $P<0.01$.

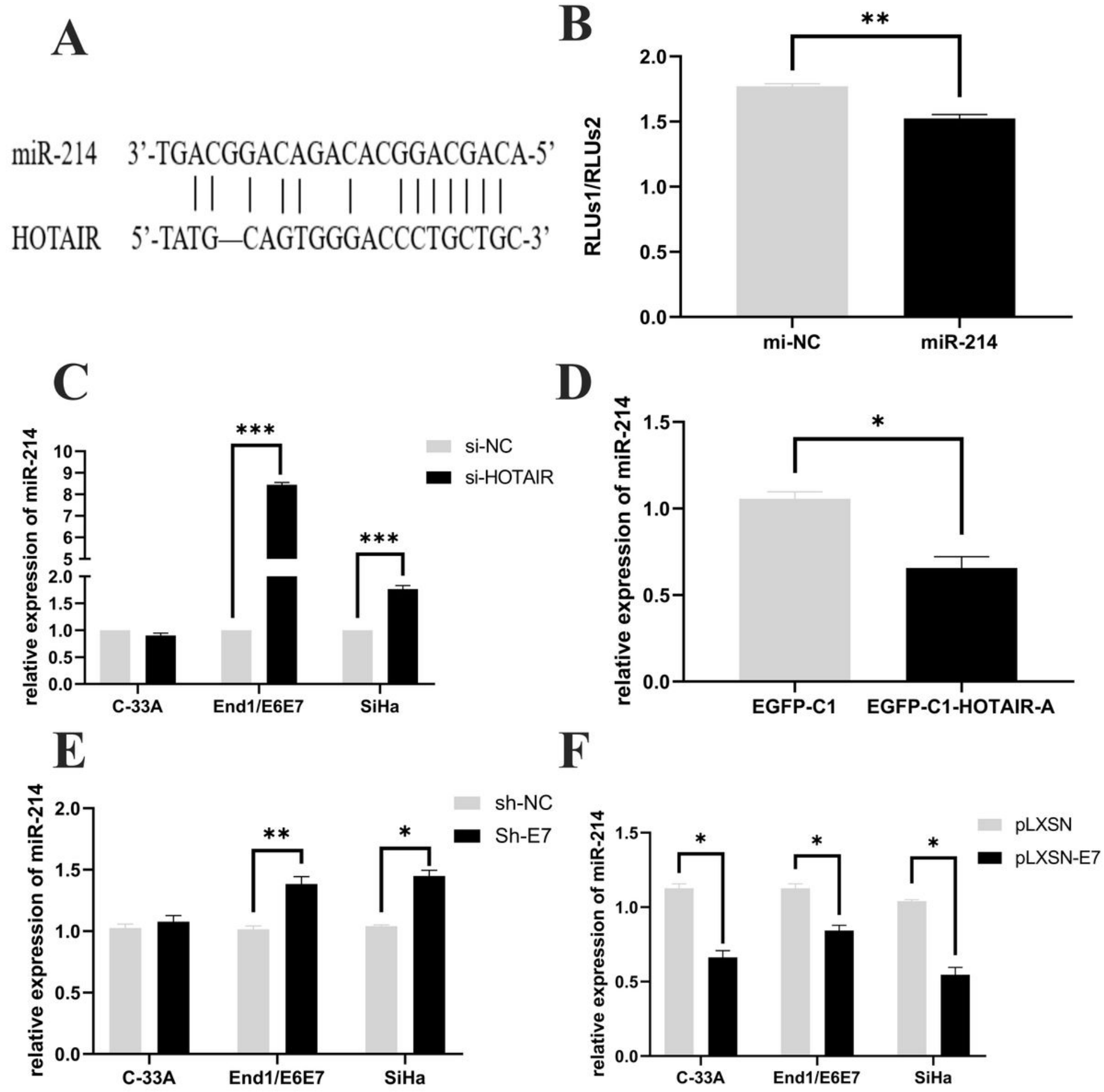




\section{Figure 4}

Validation of HOTAIR as a sponge of miR-214-3p in cervical cancer cells. (A) Bioinformatics analysis of miR-214-3p response elements on HOTAIR sequence. The seed sequences of miR-214-3p (CAGCAGG) were fully complementary to the 1807-1813 bp on HOTAIR. (B) The activity of luciferase was significantly reduced after co-transfected with miR-214-3p mimics and HOTAIR reporter plasmid in HEK-293T cells overexpressing miR-214-3p. (C) Knockdown of HOTAIR significantly upregulated the expression of miR214-3p in HPV16 positive cells, but not in HPV negative C-33A cells. (D) HOTAIR-A (contain MRE) obviously inhibited miR-214-3p expression in C-33A cells. (E\&F) The expression level of miR-214-3p was significantly upregulated or down regulated when HPV16 E7 was inhibited and overexpressed in C-33A, End1/E6E7 and SiHa cells, respectively. The mean values and standard error were obtained from three independent experiments. *: $\mathrm{P}<0.05, * *: \mathrm{P}<0.01, \star \star \star: P<0.001$.

A

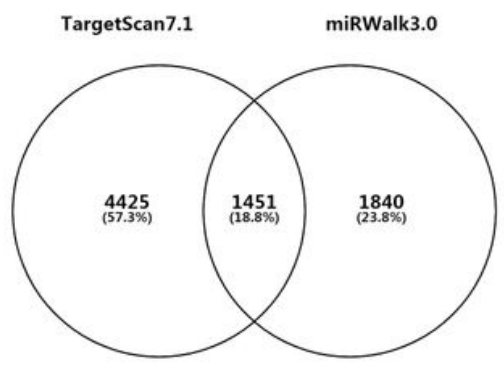

B

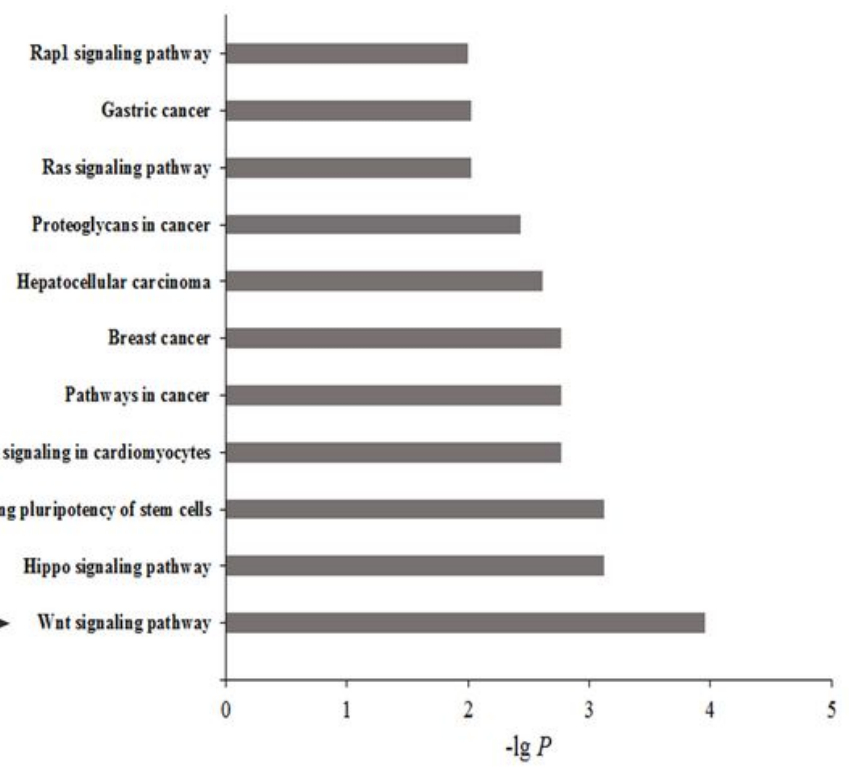

\section{Figure 5}

The signal pathway of miR-214-3p target genes was predicted with bioinformatics analysis. (A) The target genes were predicted with TargetScan7. 2 and miRWALK3.0. Totally 1451 genes were screened by two databases analysis. (B) KEGG pathway enrichment analysis was performed to screen the key pathway related with miR-214-3p. The arrow indicates the highest-ranking signaling pathway. 


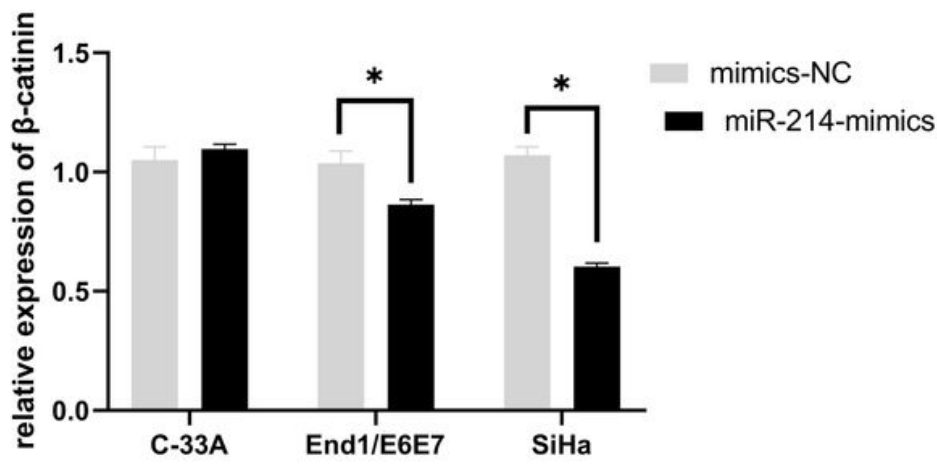

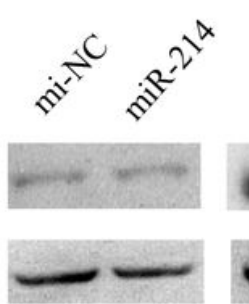

C-33A

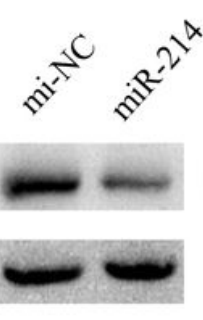

End1/E6E7

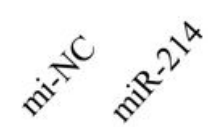

$\beta$-catenin(110kD)

$\beta-\operatorname{actin}(46 \mathrm{kD})$

$\mathrm{SiHa}$
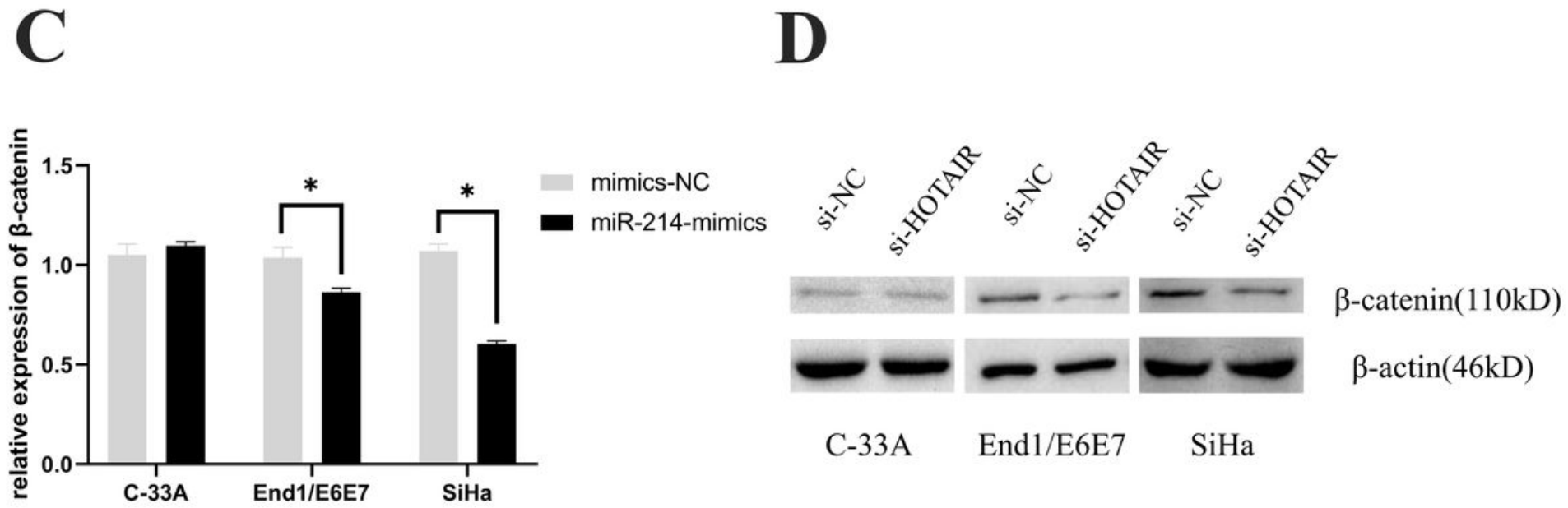

C-33A End1/E6E7 SiHa

E

F
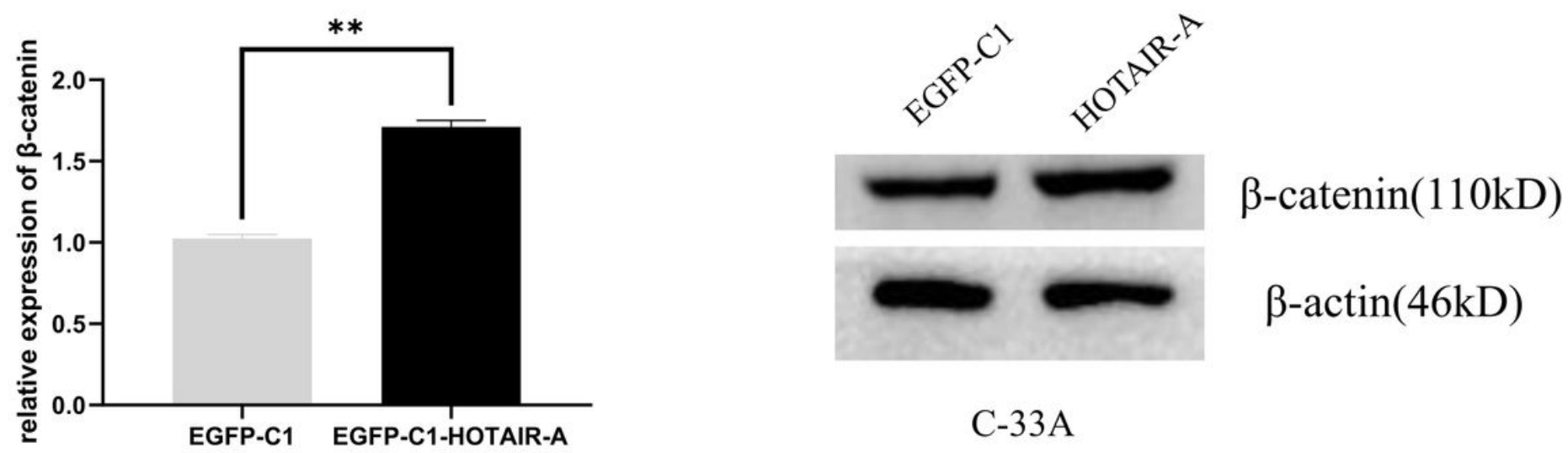

Figure 6

HOTAIR/miR-214-3 axis mediated the expression of $\beta$-catenin in HPV16 positive cervical cancer cells. (A\&B) Over-expression of miR-214-3p could inhibit the expression of $\beta$-catenin mRNA and protein by qPCR and western blotting analysis in End1/E6E7 and SiHa cells. (C\&D) Knocking down HOTAIR could inhibit the expression of $\beta$-catenin mRNA and protein by $\mathrm{qPCR}$ and western blotting analysis in End1/E6E7 and SiHa cells. (E\&F) Overexpressed of HOTAIR-A (contain MRE) obviously up-regulated the expression of $\beta$-catenin mRNA and protein in C-33A cells. 